\title{
Enhancement of optical code division multiple access system based on 2D ZCC/MD spectral/spatial code
}

\author{
Rima. Matem, S. A. Aljunid, M. N Junita, C. B.M Rashidi, Israa Shihab Aqrab \\ Advanced Communication Engineering Center of Excellence School of Computer and Communication Engineering \\ (ACE CoE-SCCE), Universiti Malaysia Perlis (UniMAP), Malaysia
}

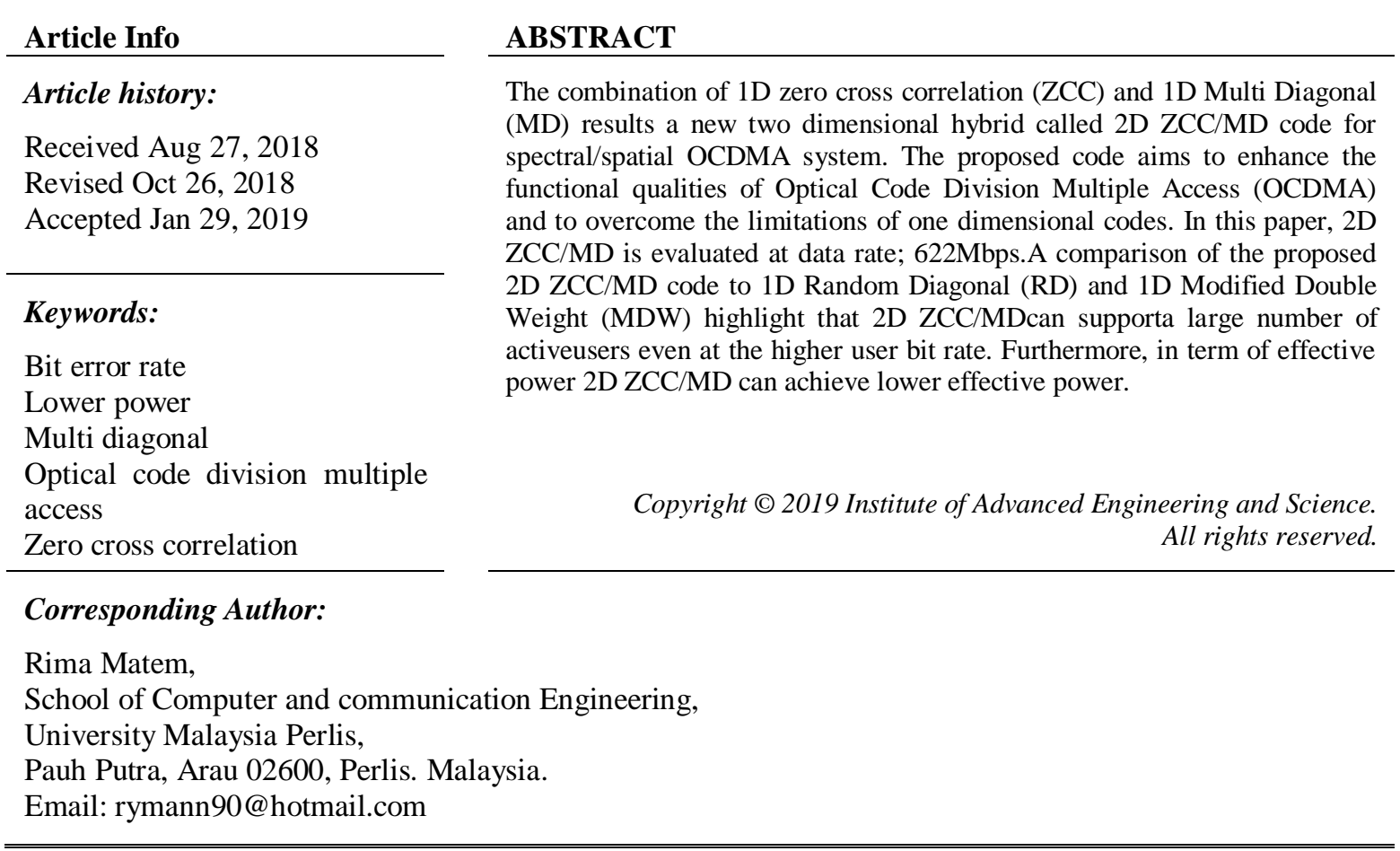

\section{INTRODUCTION}

The most popular multiple access technique nowadays is the OCDMA [1], [2], [3]. The introduction of Optical CDMA (OCDMA) brought fascination to researchers as it displayed the potential for betterment of spectral efficiency, improvement of information security, and flexibility of bandwidth [4]. The OCDMA technique enables many subscribers to share the optical network at the same time and asynchronously by allocating a specific code to reach subscriber. OCDMA is multiplexing technique, where the information bit stream of every client is optically encoded, based on a unique assigned code sequence for every client to be transmitted through optical fiber system asynchronously.

OCDMA system is able to run in one-dimensional (1-D), various schemes have been proposed for one-dimensional incoherent OCDMA are Time Spreading (TS) and Spectral Amplitude Coding (SAC-OCDMA), where the codes are implemented in the time or frequency domains, respectively [5]. In order to reduce influence of the MAI effect, the Spectral Amplitude Coding (SAC) method has been proposed,which utilizes codes with fixed in-phase cross-correlation, $\lambda c$ [6]. A large number of codes were proposed for SAC-OCDMA system such as Modified Quadratic Congruence code (MQC) [7], [8], Modified Double Weight code (MDW) [9], [10], [11], M-sequence code [12], [13] and ZCC [14], [15],flexible Cross Correlation (FCC) [16] to reduce the MAI's influence from the system. Some weaknesses have been highlighted by previous studies of 1D codes- for instance, lengthy codes and the increasing of MAI in the system [17]. Wide bandwidth sources are required to accommodate more subscribers in the network that reduce the spectral efficiency [18].

To overcome the limitations of $1 \mathrm{D}$ codes, 2D codes were proposed via the combination of two resources (time, wavelength, spatial). The 2D code approach uses shorter code length than 1D to increase the 
number of subscribers in the network by spreading the code in two domains. The $2 \mathrm{D}$ approach has the potential to reduce the effects of MAI and PIIN, accommodate many active users at high data rates and increase spectral efficiency. The present work was initiated to introduce the new 2D OCDMA coding system that can enhance the system performance of the OCDMA network. The following section, will be described the code design of 2D ZCC/MD code. Section 3 demonstrates the results and discussion. The conclusion will be the Section 4.

\section{CODE DESIGN}

The combination of 1D ZCC code [14] and 1D MD code [19] is the basis for generating the 2DZCC/MD code. Let Let $X=\left\{x_{0}, x_{1}, x_{2}, \ldots, x_{M-1}\right\}, Y=\left\{y_{0}, y_{1}, y_{2}, \ldots, y_{N-1}\right\}$ With $k_{1}, k_{2}$ code weight of 1DZCC code and1D-MD code weight respectively. Denoted that $w_{1}, w_{2}$ are the code length of 1D-ZCC code andis 1D-MD code respectively, thus the code length of $\mathrm{X}$ is $M=k_{1} w_{1}$ where $N=k_{2} w_{2}$ is the code length of $Y . W=w_{1} w_{2}$ represents the code size of 2D-ZCC/MD.

\subsection{D Zero Cross Correlation}

In 2009, Anuar et al [14] and his colleague proposed a new code for SAC-OCDMA systems, called the Zero Cross Correlation (ZCC) code. It is represented as $\left(N, w, \lambda_{c}=0\right)$, whereby, the Hamming weight can be increased for any number larger than one.

The ZCC code design is based on the MDW code family with zero cross correlation function $\left(\lambda_{c}=\right.$ $0)$ to eliminate multi access interference and reduce system complexity. However, the ZCC code can be represented by utilizing the $K x N$ matrix. To generate the ZCC code, four matrices $([A],[B],[C],[D])$ must be created to produce the basic ZCC code matrix as in [14]:

$$
Z C C_{w}=\left[\begin{array}{ll}
A & B \\
C & D
\end{array}\right]
$$

Where $[A],[B],[C]$, and $[D]$ matrices are as follows:

1. $[A]$ Comprises of $[1, w(w-1)]$ matrix of zeros.

2. $\quad[B]$ Consists of $w$ replication of the matrix $\sum_{j=1}^{w} j[0,1]$.

3. $[C]$ Comprises of the duplication of the matrix from $w-1$.

4. [D] Comprises of the diagonal pattern of $[m \times n]$ with exchange column of zeros matrix $[m \times n]$.

The equation that co-relates the basic number of users $K$, the Hamming weight $(w)$ and basic code length $L$ is as follows:

$$
\begin{aligned}
& K=w+1 \\
& L=w(w+1)
\end{aligned}
$$

An example of the ZCC for $w=3$ is shown as follow:

$A B$

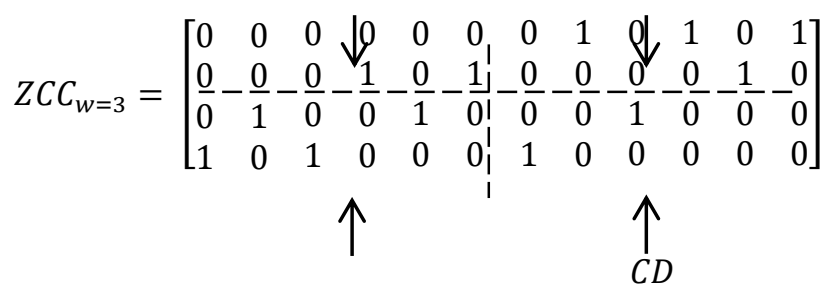

Based on the basic matrix of the ZCC code numbers of users increases by utilizing the mapping method. This method is utilized to maintain zero cross correlation between the generated code words.

\subsection{D Multi Diagonal (MD) code:}

One-dimensional Multi Diagonal (1D MD) was formulated by Thanaa et al [19]. It is characterized by $\left(N, w, \lambda_{c}\right)$ in which $N$ symbolizes (code length), $w$ represents (code weight) and $\lambda_{c}$ represents (in phase cross correlation). 
For the code sequence $X=\left\{x_{1}, x_{2}, \ldots, x_{N}\right\}, Y=\left\{y_{1}, y_{2}, \ldots, y_{N}\right\}$. The cross correlation expression can be represented by $\lambda_{c}=\sum_{i}^{N} x_{i} y_{i}$.

$1 D M D$ (Multi Diagonal) possesses zero cross correlation since $\lambda_{c}=0$. The matrix of 1D MD code comprises of $K x N$ functionality depending on value of $w$ (code weight) and $K$ (number of users). Weight value is chosen freely and $N=K w$. 1D MD code may be developed as follow:

Step1: A sequence of diagonal matrix can be formed based on the number of user $(K)$ and value of the weight $(\mathrm{w})$. Index of rows in each matrix is represented by the $i(i=1,2,3, \ldots . K)$ whereas $\operatorname{in} j(j=$ $1,2,3, \ldots ., w), \mathrm{j}$ is the number of diagonal matrix.

Step2: The MD sequences can be computed for each diagonal matrix following this equation:

$$
\begin{aligned}
S_{i, j_{w}}= & \left\{\begin{array}{r}
\left(i_{n}+1-i\right), j_{w}=\text { even } \\
i \text { for } j_{w}=\text { odd }
\end{array}\right. \\
S_{i, 1}= & {\left[\begin{array}{c}
1 \\
2 \\
3 \\
\cdot \\
\cdot \\
\cdot \\
K
\end{array}\right], S_{i, 2}=\left[\begin{array}{c}
K \\
\cdot \\
\cdot \\
\cdot \\
3 \\
2 \\
1
\end{array}\right], S_{i, 3}=\left[\begin{array}{c}
1 \\
2 \\
3 \\
\cdot \\
\cdot \\
\cdot \\
K
\end{array}\right] }
\end{aligned}
$$

Each element of the $S_{i, j}$ matrices consists the position of one in $T_{i, j}$ matrices with $K x K$ dimensions. Where

$$
\begin{aligned}
& T_{i, 1}=\left[S_{i, 1}\right]_{K \times K}, T_{i, 2}=\left[S_{i, 2}\right]_{K \times K}, \cdots, T_{i, w}=\left[S_{i, w}\right]_{K \times K} \\
& T_{i, 1}=\left[\begin{array}{cccc}
1 & 0 & \cdots & 0 \\
0 & 1 & \cdots & 0 \\
\vdots & \vdots & \ddots & \vdots \\
0 & \cdots & \cdots & 1
\end{array}\right]_{K \times K}, T_{i, 2}=\left[\begin{array}{cccc}
0 & \cdots & 0 & 1 \\
0 & \cdots & 1 & 0 \\
\cdots & \cdots & \ddots & \vdots \\
1 & \cdots & \cdots & 0
\end{array}\right]_{K \times K}, \ldots, T_{i, w}=\left[\begin{array}{cccc}
1 & 0 & \cdots & 0 \\
0 & 1 & \cdots & 0 \\
\vdots & \vdots & \ddots & \vdots \\
0 & \cdots & \cdots & 1
\end{array}\right]_{K x K}
\end{aligned}
$$

Step3: As results, the MD code is represented by the total combination of diagonal matrices as a matrix of power $K x N$.

$$
\begin{aligned}
& M D=\left\lfloor T_{i, 1} \vdots T_{i, 2} \vdots \cdots \cdots: T_{i, w}\right\rfloor_{K x N} \\
& M D=\left[\begin{array}{cccc}
a_{1,1} & a_{1,2} & \cdots & a_{1, N} \\
a_{2,1} & a_{2,2} & \cdots & a_{2,1} \\
a_{3,1} & a_{3,2} & \cdots & a_{3, N} \\
\vdots \vdots & \cdots & \vdots \\
a_{i_{n}, 1} & a_{i_{n}, 2} & \cdots & a_{i_{n}, N}
\end{array}\right]
\end{aligned}
$$

\begin{tabular}{|c|c|c|c|}
\hline$A_{g, h}$ & \multicolumn{3}{|c|}{$\left[\begin{array}{llllll}0 & 0 & 0 & 1 & 0 & 1\end{array}\right]\left[\begin{array}{lllllll}0 & 1 & 0 & 0 & 1 & 0\end{array}\right]\left[\begin{array}{llllll}1 & 0 & 1 & 0 & 0 & 0\end{array}\right]$} \\
\hline [1] & {$\left[\begin{array}{llllllll}0 & 0 & 0 & 1 & 0 & 1\end{array}\right]$} & $\begin{array}{lllllllll}0 & 1 & 0 & 0 & 1 & 0\end{array}$ & {$\left[\begin{array}{lllllll}1 & 0 & 1 & 0 & 0 & 0\end{array}\right]$} \\
\hline 0 & $\begin{array}{llllll}0 & 0 & 0 & 0 & 0 & 0\end{array}$ & $\begin{array}{lllllll}0 & 0 & 0 & 0 & 0 & 0\end{array}$ & $\begin{array}{lllllll}0 & 0 & 0 & 0 & 0 & 0\end{array}$ \\
\hline 0 & $\begin{array}{lllllll}0 & 0 & 0 & 0 & 0 & 0\end{array}$ & 0000000 & $\begin{array}{lllllll}0 & 0 & 0 & 0 & 0 & 0\end{array}$ \\
\hline & & 010010 & 101000 \\
\hline
\end{tabular}

For example, $K=4$ and $w=3$ so $N$ is equal to 12 , the MD code sequences will be:

$$
M D=\left[\begin{array}{llllllllllll}
1 & 0 & 0 & 0 & 0 & 0 & 0 & 1 & 1 & 0 & 0 & 0 \\
0 & 1 & 0 & 0 & 0 & 0 & 1 & 0 & 0 & 1 & 0 & 0 \\
0 & 0 & 1 & 0 & 0 & 1 & 0 & 0 & 0 & 0 & 1 & 0 \\
0 & 0 & 0 & 1 & 1 & 0 & 0 & 0 & 0 & 0 & 0 & 1
\end{array}\right]
$$

Example of 2-D ZCC/MD Code Sequences for $k_{1}=2$ and $k_{2}=2$ as shown in Table 1.

Table 1. Example of 2-D ZCC/MD Code Sequences for $k_{1}=2$ and $k_{2}=2$

Four characteristics may determine the cross correlation of 2D ZCC/MD code, as follow: 


$$
\begin{aligned}
& A^{0}=Y^{T} X \\
& A^{1}=Y^{T} \bar{X} \\
& A^{2}=\overline{Y^{T} X} \\
& A^{3}=\overline{Y^{T} X}
\end{aligned}
$$

Where A (d), $\mathrm{d} \in(0,1,2,3)$ represents the characteristic matrices [18]. $X$ and $Y$ is the counterpart of $\bar{X}$ and $\bar{Y}$ respectively. The following expresses the cross correlation of hybrid 2D ZCC/MD code $A^{(d)}$ and $A_{g, h}$ :

$$
\left.R^{(d)}(g, h)=\right) \sum_{M=1}^{M-1} \sum_{N=1}^{N-1} a_{i j}^{(d)} a_{(i+g)(j+h)}
$$

In which $a_{i j}^{(d)}$ consists of the $(i, j)_{t h}$ of $A^{(d)}$ and $a_{(i+g)(j+h)}$ is the $(i, j)_{t h}$ of $A_{g, h}$. The following illustrates the computation of the cross correlation of $A_{0,0}^{0}$ and $A_{g, h}$ :

$$
\begin{aligned}
& R^{(0)}(g, h)=\sum_{i=1}^{M-1} \sum_{j=1}^{N-1} a_{i, j}^{(0)} a_{i, j}(g, h)= \\
& \left\{\begin{array}{c}
k_{1} k_{2} \text { for } g=0, h=0 \\
\text { 0otherwise }
\end{array}\right.
\end{aligned}
$$

To assess the performances of the proposed 2D ZCC/MD code, the signal to noise ratio (SNR) and the bit error rate (BER) must be calculated using the following equations:

$$
S N R=I^{2} / \sigma^{2}
$$

Where I represents the incident current, expressed as:

$$
I=\Re \int_{0}^{\infty} G(v) d v
$$

And noise variance is represented by $\sigma^{2}$, which is the sum of the powers of shot, PIIN (phase induced intensity noise) and thermal noise $\sigma$ :

$$
\sigma^{2}=i_{\text {PIIN }}^{2}+i_{\text {shot }}^{2}+i_{\text {thermal }}^{2}
$$

From (14), the $\mathfrak{R}$ represents the PIN photodiode responsivity.

$$
\begin{aligned}
& \left\langle i_{\text {noise }}^{2}\right\rangle=\left\langle i_{\text {PIIN }}^{2}\right\rangle+\left\langle i_{\text {shot }}^{2}\right\rangle+\left\langle i_{\text {thermal }}^{2}\right\rangle \\
& =B I^{2} \tau+2 e B I+\frac{4 K_{b} T_{n} B}{R_{L}}
\end{aligned}
$$

$e$ : electron's charge.

$K_{b}$ : constant of Boltzmann.

$I:$ stands for the average photocurrent.

$R_{L}$ : the load resistance.

$T_{n}$ : the unlimited temperature.

$B$ : the electrical bandwidth.

The 2D ZCC/MD code system represents a property of zero cross correlation, so the impact of Multiple Access Interference has been removed. Add on, there in an equal chance of distributing bit 1 for each user. At the receiver $(0,0)$, theoutputcurrents is counted following the cross-correlation of $A_{0,0}^{(0)}$ and $A_{g, h}$ as:

$$
\begin{aligned}
& I=R \int_{0}^{\infty} G(v) d v \\
& =\frac{R P_{s r} k_{1}}{M}
\end{aligned}
$$

The formula below shows the formulations of equations for Shot noise, PIIN and Thermal noise: 


$$
\begin{aligned}
& \left\langle i_{\text {PIIN }}^{2}\right\rangle=B \frac{R^{2} P_{S r}^{2} w_{2} k_{1}}{\Delta v W} \\
& \left\langle i_{\text {shot }}^{2}\right\rangle=2 e B \frac{R P_{s r} w_{2}}{W} \\
& \left\langle i_{\text {thermal }}^{2}\right\rangle=\frac{4 K_{b} T_{n} B}{R_{L}}
\end{aligned}
$$

So:

$$
\left\langle i_{\text {noise }}^{2}\right\rangle=2 e B \frac{R P_{s r} w_{2}}{W}+\frac{R^{2} P_{s r}^{2} w_{2} k_{1}}{\Delta v W}+\frac{4 K_{b} T_{n} B}{R_{L}}
$$

Where $P_{s r}$ the effective source is power at the receiver, $R$ is the responsivity, $k_{1}$ and represent the code weight for spectral sequences and $k_{2}$ represents spatial sequences. The number of simultaneous clients, $N$ is the length of spatial code sequences and $M$ the spectral code sequences. Observation: the likelihood of delivering bit " 1 " for every user at a different time is being $1 / 2$, thus:

$$
\left\langle i_{\text {noise }}^{2}\right\rangle=\frac{e B R P_{s r} w_{2}}{W}+\frac{R^{2} P_{s r}^{2} w_{2} k_{1}}{2 \Delta v W}+\frac{4 K_{b} T_{n} B}{R_{L}}
$$

At the receiver, the SNR can be expressed as follow:

$$
S N R=\frac{I^{2}}{\left\langle i_{\text {noise }}^{2}\right\rangle}=\frac{\left[\frac{R P_{s r} w_{2}}{W}\right]^{2}}{\frac{R^{2} P_{S r}^{2} w_{2} k_{1}}{2 \Delta v W}+\frac{e B R P_{s r} w_{2}}{W}+\frac{4 K_{b} T_{n} B}{R_{L}}}
$$

From SNR the BER can be derived as shown in (29):

$$
B E R=\frac{\operatorname{erfc}(\sqrt{S N R / 8})}{2}
$$

Where

$$
\operatorname{erfc}=\frac{2}{\sqrt{\pi}} \int_{0}^{\infty} \exp \left(-y^{2}\right) d y
$$

Parameters Description as shown in Table 2.

Table 2. Parameters Description

\begin{tabular}{lc}
\hline The broadband light source's spectral width & $\Delta \lambda=300 \mathrm{~nm}(\Delta \lambda=3.75 \mathrm{THZ})$ \\
\hline PD quantum efficiency & $R=0.75$ \\
Operating wavelength & $\lambda_{\mathbf{0}}=1.55 \mu \mathrm{m}$ \\
Data transmission rate & $R_{b}=622 \mathrm{Mbps}$ \\
Electrical bandwidth & $B=320 \mathrm{MHz}$ \\
Receiver load resistor & $R_{L}=1030 \Omega$ \\
Receiver noise temperature & $T_{n}=300 \mathrm{~K}$ \\
Electron's charge & $e=1.60217646 \times 10^{-19} \mathrm{coulombs}$ \\
Constant of Boltzmann & $K_{b}=1.38 \times 10^{-23} \mathrm{~W} / \mathrm{K} / \mathrm{Hz}$ \\
\hline
\end{tabular}

\section{RESULTS AND DISCUSSIONS}

The list of parameters highlighted in the table assesses the impact of 2D ZCC MD on the OCDMA performance. The following figures show the results. Figure 1 represents the variation of BER against the number of users of the codes 2D ZCC/MD $(\mathrm{M}=62, \mathrm{~N}=3)$ in comparison to 1D MDW (N=58), 1D RD $(\mathrm{N}=50), 2 \mathrm{D} Z \mathrm{ZCC} / \mathrm{MD}(\mathrm{M}=23, \mathrm{~N}=7)$ where effective power Psr equal to $-10 \mathrm{dBm}$ and data rate $\mathrm{Rb}=622 \mathrm{Mbps}$. $2 \mathrm{D} \mathrm{ZCC/MD}$ is shown to exhibit the better performance than other codes. At BER equal to $10^{-9}$ the $2 \mathrm{D}$ ZCC/MD (M=23, N=7) can support more than 400 user. 


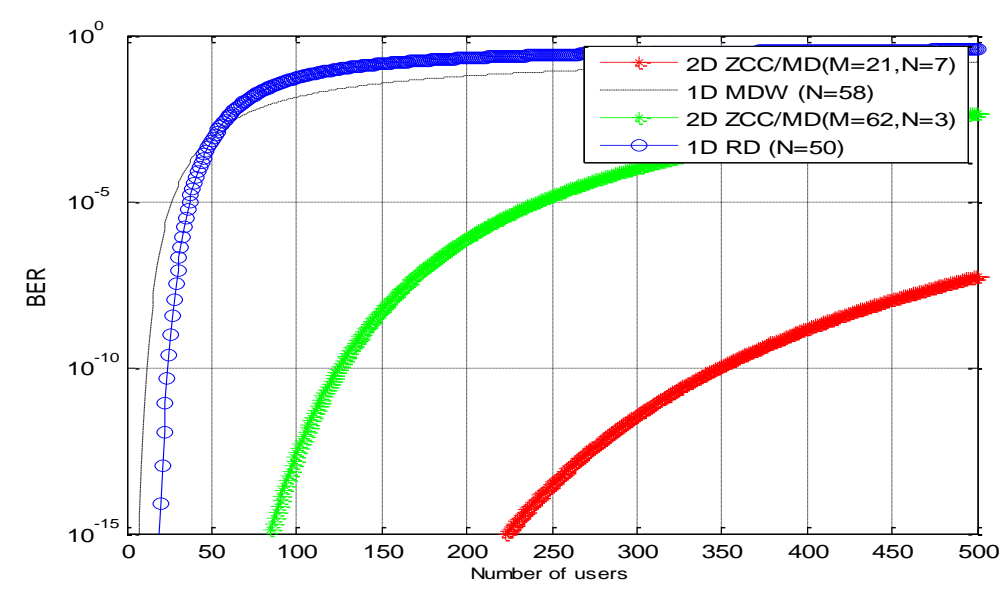

Figure 1. BER versus number of users where $\mathrm{Rb}=622 \mathrm{Mbps}$ and $\mathrm{Psr}=-10 \mathrm{dBm}$

Figure 2 shows the relation between the BER and the effective power in $\mathrm{dBm}$ when the quantity of user is equal to 100 and data rate equal to $622 \mathrm{Mbps}$. The figure apparent that $2 \mathrm{D} Z \mathrm{ZCC} / \mathrm{MD}(\mathrm{M}=63, \mathrm{~N}=3)$ is the best at high power and it has a minimum BER -24 comparing to the other codes. 2D ZCC/MD indicates that the superior performance is coming from the ability of the code to fully hinder the impact of the MAI and mitigate PIIN.

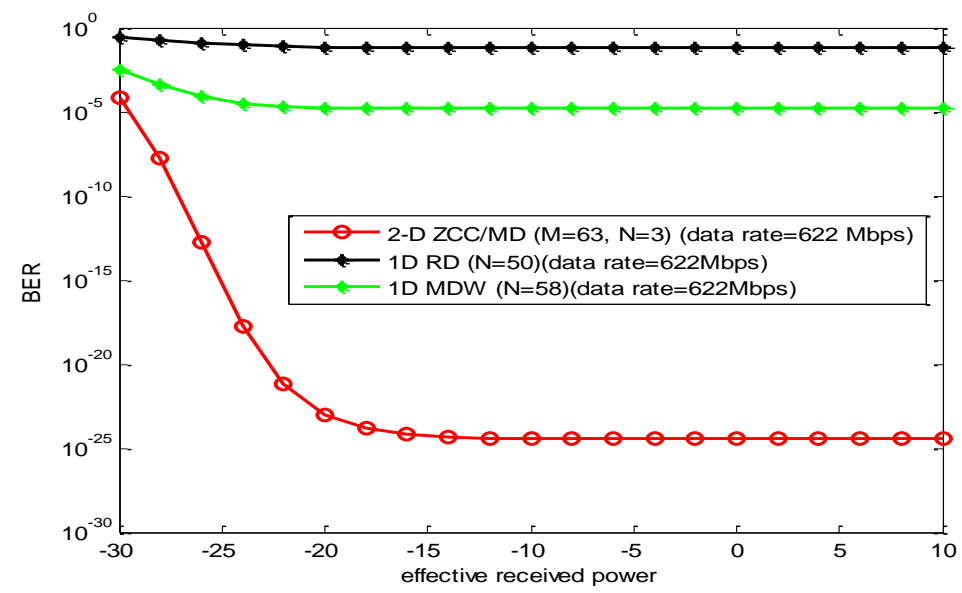

Figure 2. BER versus effective power Psr when $k=100$ user and data rate is $R b=622 \mathrm{Mbps}$

\section{CONCLUSION}

A suggested code called 2D ZCC/MD is evaluated and studied in terms of number of users and effective power. The code is constructed by combining 1D MD for spatial spreading and 1D ZCC for spectral encoding. The current study explores the comparison between one dimensional codes and two dimensional codes using 2D ZCC/MD (M=63, N=3) in comparison to 1D MDW (N=50) and 1D RD $(\mathrm{N}=58)$. According to the numerical results, the performance of $2 \mathrm{D} \mathrm{ZCC} / \mathrm{MD}$ is more favorable in terms of number of users where it is able to accommodate a significant number of active users. Moreover, a lower effective power is also can be

\section{ACKNOWLEDGEMENTS}

The author would like to acknowledge the support from Fundamental Research Grant Scheme (FRGS) under a grand number FRGS /2/2014/ICT06/UNIMAP/03/1, From the Ministry of Education Malaysia. 


\section{REFERENCES}

[1] Fadhil, H. A., Aljunid, S. A., Ahmed, H. Y., \& AlKhafaji, H. M. (2012). Variable cross-correlation code construction for spectral amplitude coding optical CDMA networks. Optik-International Journal for Light and Electron Optics, 123(11), 956-963.

[2] Eltaif, T., Shalaby, H. M., Shaari, S., \& Hamarsheh, M. M. (2009). Performance analysis of a hybrid fiber Bragg grating-based spectral-amplitude-coding/successive interference cancellation for optical CDMA systems. Optics Communications, 282(1), 1-6.

[3] Rana, S., \& Gupta, A. (2016). A Novel Security Enhanced Decoder Based on XOR Detection for Optical Code Division Multiple Access System Using Multi-Diagonal Code. Indian Journal of Science and Technology, 9(36).

[4] Kaur, N., Goyal, R., \& Rani, M. (2017). A Review on Spectral Amplitude Coding Optical Code Division Multiple Access. Journal of Optical Communications, 38(1), 77-85.

[5] Kadhim, R. A., Fadhil, H. A., Aljunid, S. A., \& Razalli, M. S. (2015). PERFORMANCE ENHANCEMENT OF A THREE DIMENSIONAL OCDMA SYSTEMS BASED ON A NEW CODE. Journal of Theoretical and Applied Information Technology, 81(3), 589.

[6] Nordin, J. M., Aljunid, S. A., Safar, A. M., Abdullah, A. R. J., \& Rahim, R. A. (2012). Performance of Hybrid Subcarrier Multiplexing Over optical CDMA Network Based on Zero Cross Correlation code”. Journal of Communication and Information Sciences (JCIS), 2(1.4).

[7] Wei, Z., \& Ghafouri-Shiraz, H. (2002). Codes for spectral-amplitude-coding optical CDMA systems. Journal of lightwave technology, 20(8), 1284.

[8] Shi, F., \& Ghafouri-Shiraz, H. (2016). Performance Analysis of Two New Code Families for Spectral-AmplitudeCoding Optical CDMA Systems. Journal of Lightwave Technology, 34(17), 4005-4014.

[9] Aljunid, S. A., Ismail, M., Ramli, A. R., Ali, B. M., \& Abdullah, M. K. (2004). A new family of optical code sequences for spectral-amplitude-coding optical CDMA systems. IEEE photonics technology letters, 16(10), 2383-2385.

[10] Aljunid, S. A., Zan, Z., Anas, A., Barirah, S., \& Abdullah, M. (2004). A new code for optical code division multiple access systems. Malaysian Journal of Science, 17(2), 30-39.

[11] Salah, M., \& Alhassan, A. M. (2017). Evaluation of Different Codes in SAC-CDMA System. Evaluation, $3(1), 34-37$.

[12] Huang, J. F., \& Chang, Y. T. (2006, May). Incoherent hybrid spectral polarization and amplitude coding implemented with specified orthogonal ternary code over balanced photo-detectors. In Communication Networks and Services Research Conference, 2006. CNSR 2006. Proceedings of the 4th Annual (pp. 8-pp). IEEE.

[13] Moghaddasi, M., Mamdoohi, G., Noor, A. S. M., Hitam, S., \& Anas, S. B. A. (2016). Evaluation of Optical Code Division Multiple Access (OCDMA) Encoding Techniques for Free Space Optics (FSO). Lasers in Engineering (Old City Publishing), 33.

[14] Anuar, M. S., Aljunid, S. A., Saad, N. M., \& Hamzah, S. M. (2009). New design of spectral amplitude coding in OCDMA with zero cross-correlation. Optics Communications, 282(14), 2659-2664.

[15] Sarma, A., \& Sarma, K. K. (2016, December). Chaotic sequence generation for a class of stochastic wireless channels. In Accessibility to Digital World (ICADW), 2016 International Conference on (pp. 121-124). IEEE.

[16] Keraf, N. D., Aljunid, S. A., Ehkan, P., \& Safar, A. M. (2016). ANALYSIS OF TWO DIMENSIONAL WAVELENGTH/TIME FCC-MDW CODE IN OPTICAL CDMA SYSTEM. Journal of Theoretical and Applied Information Technology, 94(1), 95.

[17] Rashidi, C. B. M., Aljunid, S. A., Ghani, F., Fadhil, H. A., \& Anuar, M. S. (2012). Cardinality Enhancement Using Flexible Cross Correlation (FCC) Code for Spectral Amplitude Coding Optical Code Division Multiple Access Systems. Journal of Applied Sciences Research, 8(12), 5614-5626.

[18] Kadhim, R. A., Fadhil, H. A., Aljunid, S. A., \& Razalli, M. S. (2014). A new two dimensional spectral/spatial multi-diagonal code for noncoherent optical code division multiple access (OCDMA) systems. Optics Communications, 329, 28-33.

[19] Abd, T. H., Aljunid, S. A., Fadhil, H. A., Ahmad, R. A., \& Saad, N. M. (2012). Design And Simulation A New Code With Zero Cross-Correlation For SAC-OCDMA Networks. Australian Journal of Basic and Applied Sciences, 6(3), 112-119. 